Research Paper

\title{
Overexpression of ZNF460 predicts worse survival and promotes metastasis through JAK2/STAT3 signaling pathway in patient with colon cancer
}

\author{
Tengfei Hao ${ }^{1,2^{*}}$, Jiannan $\mathrm{Xu}^{1,2^{*}}$, Sufen Fang${ }^{1}$, Jianlong Jiang ${ }^{1}$, Xinyuan Chen ${ }^{1}$, Wenhui $\mathrm{Wu}^{1}$, Liang $\mathrm{Li}^{1}$, \\ Mingzhe Li ${ }^{1}$, Changhua Zhang ${ }^{\circledR}$ and Yulong $\mathrm{He}^{1,2 \llbracket}$ \\ 1. Digestive Disease Center, The Seventh Affiliated Hospital of Sun Yat-sen University, Shenzhen, Guangdong, 518107, China. \\ 2. Department of Gastrointestinal Surgery, The First Affiliated Hospital of Sun Yat-sen University, Guangzhou, 510000, China. \\ *These authors have contributed equally to this work.
}

$\triangle$ Corresponding authors: Yulong He, MD, PhD. Digestive Disease center, The Seventh Affiliated Hospital of Sun Yat-sen University, No.628, Zhenyuan Rd, Guangming (New) Dist., Shenzhen, China. Department of Gastrointestinal Surgery, The First Affiliated Hospital of Sun Yat-sen University, Guangzhou, 510000, China. E-mail: heyulong@mail.sysu.edu.cn; Changhua Zhang, MD, PhD. Digestive Disease Center, The Seventh Affiliated Hospital of Sun Yat-sen University, No.628, Zhenyuan Rd, Guangming (New) Dist., Shenzhen, China. E-mail: zhchangh@mail.sysu.edu.cn.

(C) The author(s). This is an open access article distributed under the terms of the Creative Commons Attribution License (https://creativecommons.org/licenses/by/4.0/). See http://ivyspring.com/terms for full terms and conditions.

Received: 2020.10.27; Accepted: 2021.02.25; Published: 2021.04.02

\begin{abstract}
Zinc finger proteins (ZNFs) are a class of protein containing zinc finger domains, and they play an important role in tumor progression. However, as a member of the ZNFs family, the effect of ZNF460 in colon cancer remains unclear. In this study, we found that the expression of ZNF460 protein were markedly increased in clinical colon cancer tissues compared with para-cancer non-cancerous tissues by tissue immunohistochemistry (IHC) and western blot (WB). We also confirmed this result at the mRNA and protein levels of ZNF460 through bioinformatics analysis. In addition, high expression of ZNF460 was correlated with increased depth of invasion $(P<0.05)$, increased lymph node metastasis $(P<0.05)$, distant metastasis $(P<0.05)$ and high blood serum CA19-9 level $(\mathrm{P}<0.05)$. High expression of ZNF460 predicted poor overall survival (OS) and recurrence free survival (RFS) in patients with colon cancer. Moreover, multivariate analyses revealed that ZNF460 was an independent prognostic factor in both OS (hazard ratio [HR]: 1.636; 95\% confidence interval [Cl], 1.028-2.603; $\mathrm{P}=0.038$ ) and RFS (HR: 2.215; 95\% Cl: 1.227-3.997; P = 0.008). The knockdown of ZNF460 suppressed the invasion and metastasis of colon cancer cells in vitro. Mechanistically, we revealed that ZNF460 promotes the activation of the JAK2/STAT3 signaling pathway in colon cancer cells. Taken together, overexpression of ZNF460 predicted worse survival and promoted metastasis through JAK2/STAT3 signaling pathway in patient with colon cancer, and could be a novel therapeutic target in colon cancer.
\end{abstract}

Key words: ZNF460; colon cancer; prognostic; metastasis; JAK2/STAT3

\section{Introduction}

Colon cancer is one of the most common types of malignant tumors and ranks fourth in terms of incidence and fifth in terms of mortality worldwide. It is the second most common malignant tumor diagnosed in women and third most in men. The highest colon cancer incidence rates are found in parts of Europe and Australia/New Zealand [1, 2]. Currently, surgery is still the most potent curative method for colon cancer $[3,4]$. But metastasis and recurrence rates after radical resection of colon cancer remained high [5]. Furthermore, some colon cancer patients were diagnosed at advanced stages with distant metastasis, which meant missing the window of surgery, thus was associated with shorter survival time and poorer prognosis [6, 7]. However, the underlying mechanisms of colon cancer metastasis remain unclear [8]. Thus, it is important to investigate the molecular mechanisms involved in colon cancer progression and metastasis.

Zinc finger proteins $(\mathrm{ZNFs})$ are a group of proteins containing zinc finger domains. ZNFs have a wide range of molecular functions and were encoded by $2 \%$ of all human genes [9]. The Zinc finger structure of protein can bind with gene promoter 
region and plays an important role in regulating cell proliferation, differentiation, apoptosis and so on [10]. ZNFs play an important role in cancer progression or tumor suppression. Previous study has revealed that ZNF306 [11], ZNF304 [12], and ZEB1 [13] contribute to tumorigenesis in colorectal cancer, but ZNF545, as a tumor suppressor, inhibits colon cancer progression [14]. ZNF460 as a C2H2-type ZNF, encoding a 562amino-acid polypeptide, was first identified in 2003 but its function in colon cancer was still unclear [15].

In this study, we first demonstrated that ZNF460 is upregulated in colon cancer tissues compared with para-cancer non-cancerous tissues. High expression of ZNF460 represented poor prognosis and might be associated with metastasis of colon cancer. Multivariate analyses revealed that ZNF460 was an independent prognostic factor in patients with colon cancer. In addition, we also provided the first evidence that ZNF460 promoted the invasion and metastasis of colon cancer cells through activating the JAK2/STAT3 signaling pathway and might be a novel therapeutic target in colon cancer.

\section{Materials and Methods}

\section{Patients}

Surgically treated colon cancer patients $(n=262)$ with confirmed pathology without neoadjuvant chemotherapy at the First Affiliated Hospital of Sun Yat-sen University between 2008 and 2011 were randomly chosen. TNM staging was performed according to the $8^{\text {th }}$ edition of American Joint Committee on Cancer Staging Manual. This study was performed according to the Declaration of Helsinki. Research ethics were approved by the ethical committee of the Seventh Affiliated Hospital of Sun Yat-sen University and the samples were obtained with informed consent.

\section{Cell culture, siRNAs and reagents}

The human colon cancer cell lines SW480, LoVo, DLD-1 and HCT-116 were purchased from the Shanghai Institute of Cell Biology, China. The LS174T cell line was purchased from the ATCC. These cells were cultured in RPMI-1640 medium supplemented with 10\% fetal bovine serum (Hyclone, Logan, UT, USA) and antibiotics at $37{ }^{\circ} \mathrm{C}$ with $5 \% \mathrm{CO}_{2}$. Colon cancer cells were transfected with either siZNF460 or empty vector siRNA (Ribo, China) using a riboFETCTM CP transfection reagent (Ribo, China) according to the manufacturer's instructions. The siRNA-transfected colon cancer cells were cultivated at $37^{\circ} \mathrm{C}$ for 48 hours. The transfected cells were divided into three experimental groups: Control, NC-siRNA, ZNF460siRNA. After 48 hours, the transfected cells were collected for protein expression analysis. The siRNA sequence is listed as follows: ZNF460siRNA\#1: 5'-GCACAGATCTCATTCAAC-3'; ZNF460siRNA\#2: 5'-GTCCCAAGATACTCCTATT-3'; ZNF460siRNA\#3: $\quad$ 5'- GCCTTCAATTGCCGCT CAT-3'.

\section{Immunohistochemistry}

Paraffin-embedded colon cancer specimens were obtained from the Department of Pathology of First Affiliated Hospital of Sun Yat-sen University. IHC staining were conducted using an anti-ZNF460 antibody (1:200; Sigma-Aldrich, Darmstadt, Germany). Immunoreactivity scores were evaluation by two independent investigators who were blinded to the clinical data of the study and used semiquantitative method to evaluated the scoring. A final agreement between them was obtained for each score, even for discrepant immunostaining results. Histochemistry score (H-SCORE) scoring method was adopted [16]. H-SCORE is a scoring method for tissue immunohistochemical results that reflects the positive ratio and the positive intensity. The following formula was used: $\mathrm{H}-\mathrm{SCORE}=\sum(\mathrm{PI} \times \mathrm{I})=$ (percentage of cells of weak intensity $\times 1)+$ (percentage of cells of moderate intensity $\times 2)+$ (percentage of cells of strong intensity $\times 3$ ). In the formula, PI represents the percentage of positive cells to the total number of cells in this position and I represent the intensity of staining. I=0: blue staining, I=1: light yellow staining, $\mathrm{I}=2$ : brown staining and $\mathrm{I}=3$ : dark brown staining. The H-SCORE is 0-300, with a higher score representing stronger positive staining.

\section{Cell migration and invasion assay}

For the migration assay, $5 \times 10^{4}$ LoVo cells and $1 \times 10^{5}$ LS174T cells were resuspended in serum-free medium and placed in the upper chambers. For the invasion assays, $1 \times 10^{5}$ LoVo cells and $2 \times 10^{5}$ LS174T cells were seeded in a Matrigel-coated chamber (BD Biosciences, Bedford, MA, USA). After 36h (to examine migration) or $48 \mathrm{~h}$ (to examine invasion) of incubation, the non-migrated cells on the upper surface of the membrane were removed, and the cells on the lower surface were fixed and stained with $0.1 \%$ crystal violet. The cells in five random microscopic fields were counted and imaged using a light microscope with Leica Microsystems DM4B.

\section{Bioinformatics analysis}

Colon cancer RNA-Seq data and protein expression data were obtained from TCGA data through the UALCAN website (http://ualcan.path. uab.edu) [17]. The prognostic role of ZNF460 mRNA levels were analyzed using the TCGA data through the following website (http://bioinfo.henu.edu.cn/ DatabaseList.jsp). ZNF460 co-expression was 
analyzed using Pearson's correlation coefficient, presenting in heatmaps, or scatter plots by using LinkedOmics database [18]. Function module of LinkedOmics performed analysis of Gene Ontology biological process (GO_BP) and KEGG pathways with gene set enrichment analysis (GSEA). The rank criterion was FDR $<0.05$ and 1,000 simulations were performed.

Table 1. Associations of ZNF460 expression with clinical parameters in 214 colon cancer patients

\begin{tabular}{|c|c|c|c|c|}
\hline \multirow[t]{2}{*}{ Characteristic } & \multirow[t]{2}{*}{ No. } & \multicolumn{2}{|c|}{ ZNF460 expression } & \multirow[t]{2}{*}{$P$ value } \\
\hline & & Low $(\mathrm{N}=107)$ & High $(\mathrm{N}=107)$ & \\
\hline Age (year) & & $58.0 \pm 14.0$ & $59.3 \pm 12.3$ & 0.584 \\
\hline$\leq 60 y$ & 101 & 53 & 48 & \\
\hline$>60 y$ & 113 & 54 & 59 & \\
\hline Gender & & & & 1.000 \\
\hline Male & 118 & 59 & 59 & \\
\hline Female & 96 & 48 & 48 & \\
\hline Tumor location & & & & 0.681 \\
\hline Right hemicolon & 98 & 47 & 51 & \\
\hline Left hemicolon & 116 & 60 & 56 & \\
\hline Differentiation & & & & 0.738 \\
\hline Well+ Moderate & 169 & 83 & 86 & \\
\hline Poor & 45 & 24 & 21 & \\
\hline Depth of invasion & & & & 0.006 \\
\hline $\mathrm{T} 1$ & 2 & 1 & 1 & \\
\hline $\mathrm{T} 2$ & 20 & 15 & 5 & \\
\hline T3 & 116 & 61 & 55 & \\
\hline $\mathrm{T} 4$ & 76 & 30 & 46 & \\
\hline Lymph node metastasis & & & & 0.025 \\
\hline No & 120 & 69 & 51 & \\
\hline $\mathrm{N} 1$ & 65 & 25 & 40 & \\
\hline N2 & 29 & 13 & 16 & \\
\hline Distant metastasis & & & & 0.039 \\
\hline M0 & 180 & 96 & 84 & \\
\hline M1 & 34 & 11 & 23 & \\
\hline TNM stage & & & & 0.001 \\
\hline I & 22 & 16 & 6 & \\
\hline II & 84 & 48 & 36 & \\
\hline III & 74 & 32 & 42 & \\
\hline IV & 34 & 11 & 23 & \\
\hline CEA level $(\mu \mathrm{g} / \mathrm{L})$ & & & & 0.126 \\
\hline$\leq 5$ & 126 & 69 & 57 & \\
\hline$>5$ & 88 & 38 & 50 & \\
\hline CA19-9 level (U/ml) & & & & 0.048 \\
\hline$\leq 35$ & 176 & 94 & 82 & \\
\hline$>35$ & 38 & 13 & 25 & \\
\hline Vessel or nerve invasion & & & & 0.147 \\
\hline Yes & 37 & 14 & 23 & \\
\hline No & 177 & 93 & 84 & \\
\hline Chemotherapy or not & & & & 0.055 \\
\hline Yes & 109 & 47 & 62 & \\
\hline No & 105 & 60 & 45 & \\
\hline
\end{tabular}

\section{Statistical analyses}

$X^{2}$ test, Wilcoxon test and $t$ test was applied for the continuous and discrete data analysis. The associations between ZNF460 expression and overall patient survival were estimated using univariate analysis and the Kaplan-Meier method and further assessed using the log-rank test. Potential prognosis and clinicopathological characteristics were adjusted for using Cox regression models of multivariate analysis, with ZNF460 expression fitted as an indicator variable. All statistical analyses were conducted using the SPSS statistical software (version 22.0; SPSS Inc., Chicago, IL) and GraphPad Prism (version 8.0). All statistical tests were two-sided, and values of $\mathrm{p}<0.05$ were considered statistically significant.

\section{Results}

\section{ZNF460 expression is upregulated in colon cancer tissues}

Unpaired colon cancer and normal tissues from TCGA indicated ZNF460 mRNA in colon cancer markedly upregulated expression ( $\mathrm{P}<0.001$; Fig. 1A). Further analysis of the expression according to tumor stage showed that ZNF460 mRNA were significantly overexpressed in all the stages $(\mathrm{P}<0.05$; Fig. $1 \mathrm{~B})$. Consistent with the mRNA expression, data from CPTAC showed that ZNF460 protein expression were also significantly upregulated in colon cancer compared to normal samples ( $\mathrm{P}<0.01$; Fig. $1 \mathrm{C}$ and Fig. 1D). Further to verify the expression of ZNF460 in colon cancer tissues, we examined ZNF460 expression in 48 paired colon cancer tissue samples and the corresponding adjacent tissues by IHC and WB (1:500; Proteintech, Wuhan, China). The IHC results showed that ZNF460 protein of $79.2 \%(38 / 48)$ patients was highly expressed in the colon cancer tissue samples (Fig. 1E and Fig. 1F). Similarly, WB results also showed the significantly elevated ZNF460 protein levels in the tissues (Fig. 1G), which was consistent with the IHC results. These results indicated that the ZNF460 expression levels were significantly upregulated in colon cancer tissues.

\section{Relationship between ZNF460 expression and clinical parameters in patient with colon cancer}

To further examine the ZNF460 expression and its clinicopathological characteristics in colon cancer, we performed IHC staining in 214 paraffin-embedded colon cancer specimens. The ZNF460 protein was mainly expressed in the cytoplasm and nucleus based on IHC results. The patients were divided into high expression group and low expression group with median $(\mathrm{H}$ score $=95)$ as the cut-off value. The associations of ZNF460 expression with clinical parameters were summarized in Table 1 . As shown in the table, ZNF460 expression level was significantly associated with the increased depth of invasion $(\mathrm{P}<0.05)$, increased lymph node metastasis $(\mathrm{P}<0.05)$, distant metastasis $(\mathrm{P}<0.05)$, high $\mathrm{CA19-9}$ level $(\mathrm{U} / \mathrm{ml})$ $(\mathrm{P}<0.05)$ and $\mathrm{TNM}$ stage $(\mathrm{P}<0.05)$. These results 
indicated that the high expression of ZNF460 may be related to the metastasis of colon cancer.

\section{High expression of ZNF460 predicted worse survival in patients with colon cancer}

To evaluate the prognostic role of ZNF460 expression, we analyzed the prognostic data of ZNF460 using TCGA. As shown in Fig. 2B, the OS were significantly worse in the high ZNF460 group compared to low ZNF460 group (Fig. 2B, log rank $\mathrm{P}=0.0039$ ). Next, the prognostic value was assessed with Kaplan-Meier analysis of ZNF460 protein expression in our cohort. The follow-up period of the
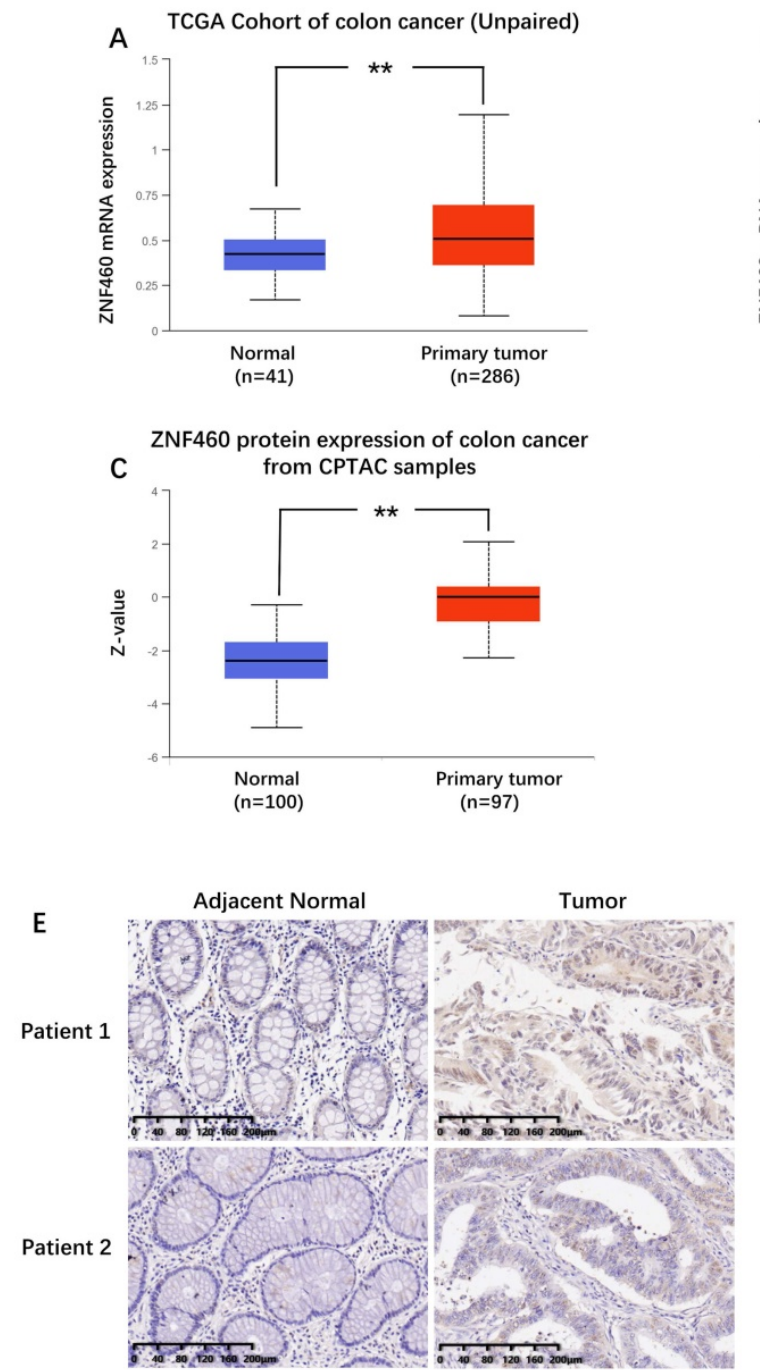

F
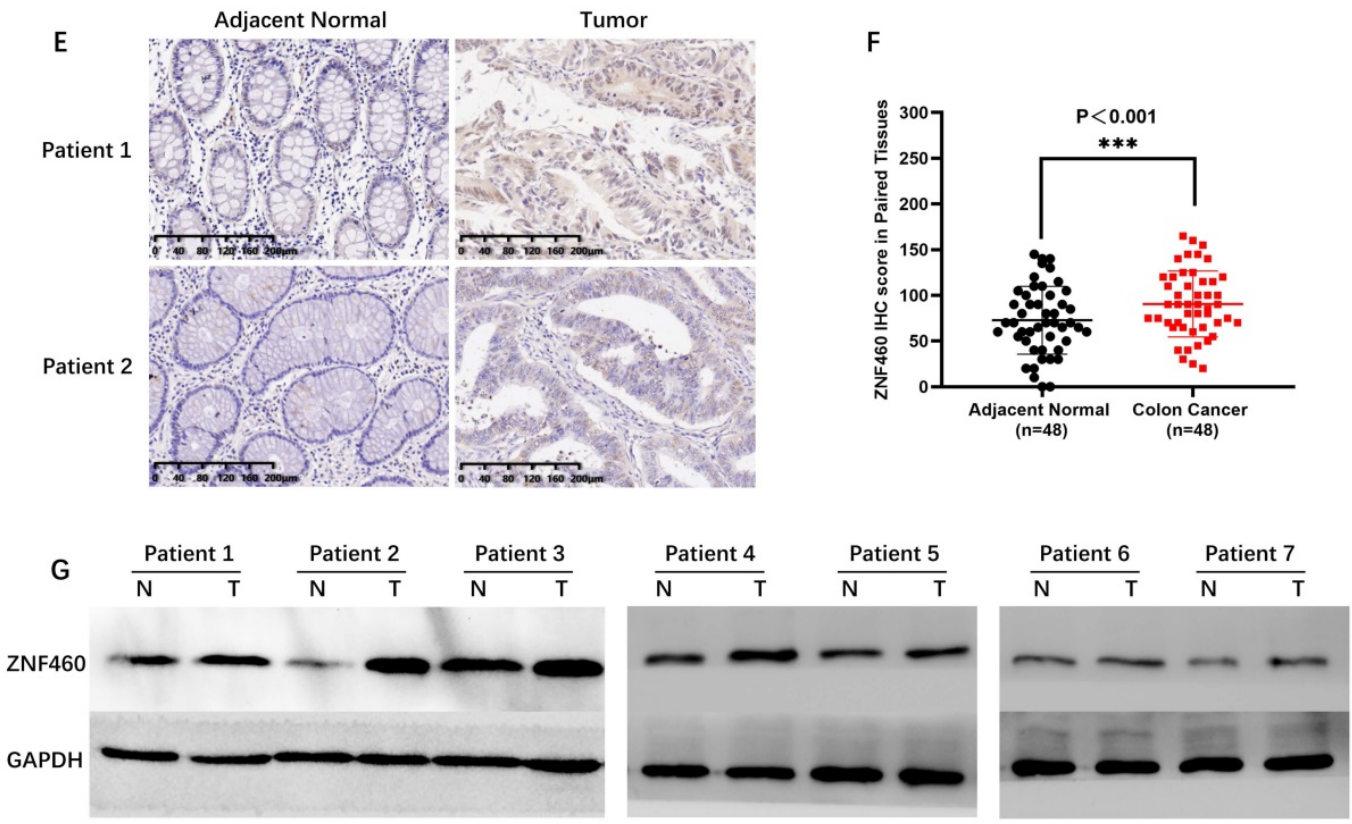

Figure 1. ZNF460 expression is upregulated in colon cancer tissues. (A,B) ZNF460 mRNA expression in unpaired colon cancer and normal tissues from the TCGA database. $* \mathrm{P}<0.05$, ** $\mathrm{P}<0.01$. (C)(D) ZNF460 protein expression in colon cancer and normal tissues from the CPTAC database. ${ }^{*} \mathrm{P}<0.01$. ZNF460 expression in 48 paired tissues by IHC $(\mathrm{E}, \mathrm{F})$ and western blotting $(\mathrm{G})$. $* * * \mathrm{P}<0.001$

214 colon cancer patients ranged from 1 to 127 months, with a mean survival time of 65 months. The results outlined that high expression of ZNF460 predicted worse OS (Fig. 2C, log rank $\mathrm{P}=0.002$ ) and RFS (Fig. 2D, log rank $\mathrm{P}=0.003$ ) in colon cancer patient. The 5-year OS rate was $76.6 \%$ in the low ZNF460 expression group and $57.9 \%$ in the high ZNF460 expression group, consistent with the TCGA data. The mean survival times of patients with low and high ZNF460 expression were 74 and 57 months, respectively.

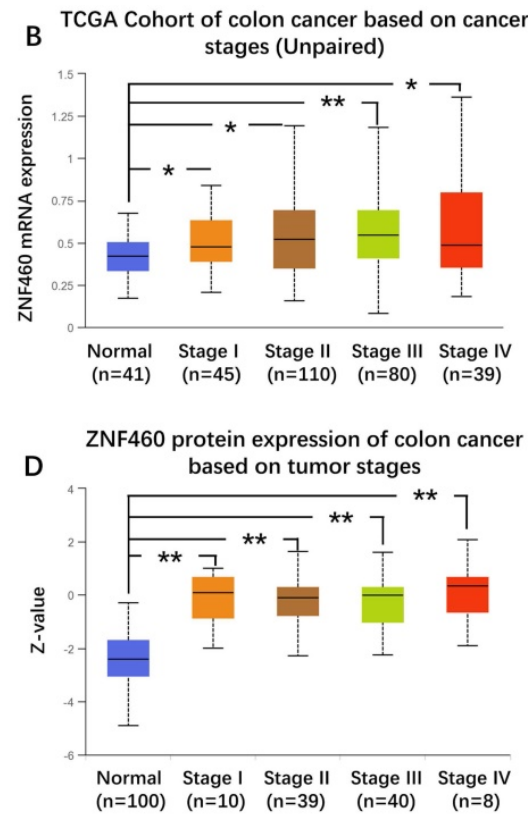


A

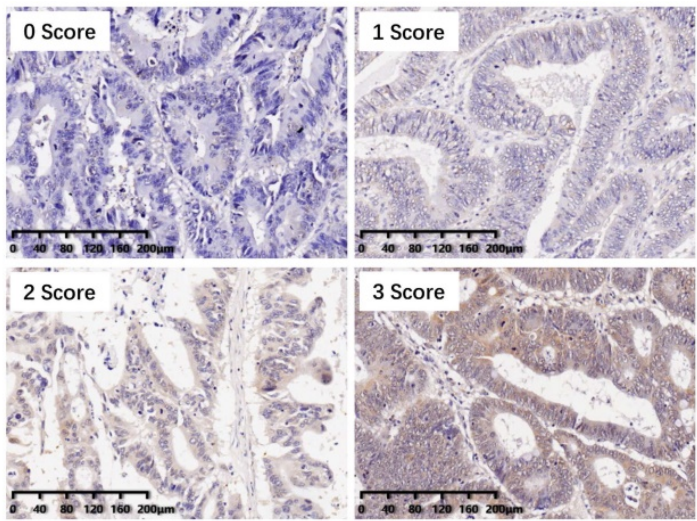

C

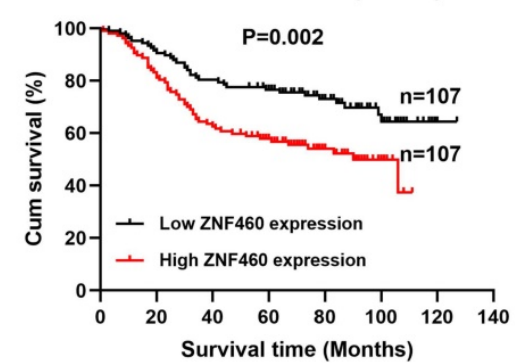

B Overall Survival of TCGA Patients $(n=453)$
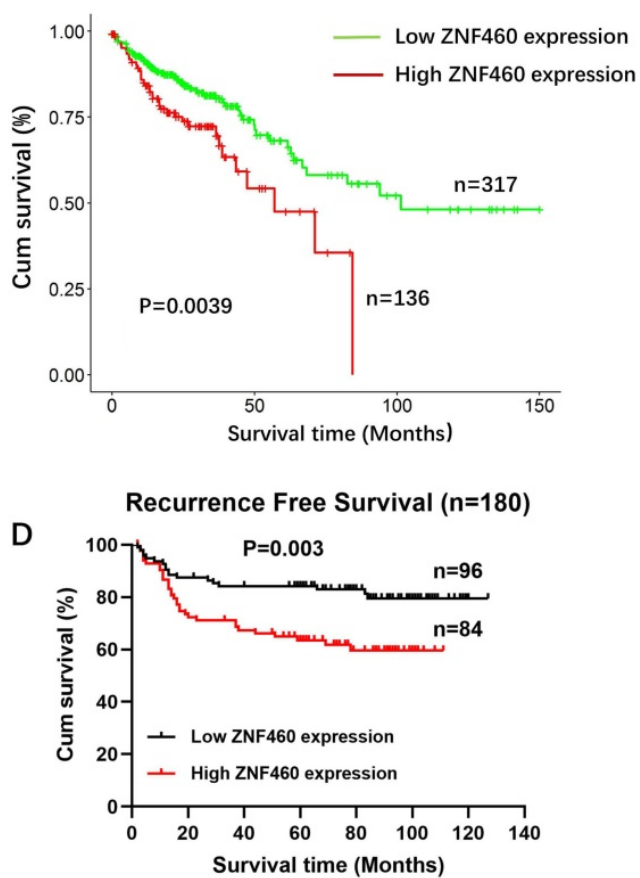

Figure 2. High expression of ZNF460 predicted worse survival in patients with colon cancer. (A) IHC staining, I=0: blue staining, I=1: light yellow staining, I=2: brown staining and $\mathrm{I}=3$ : dark brown staining. (B) OS from TCGA database. Log rank $P=0.0039$. (C) OS from our cohort. Log rank $P=0.002$. (D) $R F S$ from our cohort. Log rank $P=0.003$.

Table 2. Cox proportional-hazard regression analysis for Overall Survival in 214 patients with colon cancer

\begin{tabular}{|c|c|c|c|c|c|c|c|c|}
\hline \multirow[t]{3}{*}{ Characteristic } & \multicolumn{4}{|c|}{ Univariate analysis } & \multicolumn{4}{|c|}{ Multivariate analysis } \\
\hline & \multirow{2}{*}{\multicolumn{2}{|c|}{$P$-value HR }} & \multicolumn{2}{|c|}{$\begin{array}{l}95.0 \% \text { CI for } \\
\operatorname{Exp}(\mathrm{B})\end{array}$} & \multirow{2}{*}{\multicolumn{2}{|c|}{$P$-value HR }} & \multicolumn{2}{|c|}{$\begin{array}{l}95.0 \% \text { CI for } \\
\operatorname{Exp}(\mathrm{B})\end{array}$} \\
\hline & & & Lower & Upper & & & Lower & Upper \\
\hline Gender & 0.971 & 1.008 & 0.654 & 1.553 & & & & \\
\hline Age & 0.911 & 0.976 & 0.634 & 1.502 & & & & \\
\hline $\begin{array}{l}\text { Tumor } \\
\text { location }\end{array}$ & 0.243 & 0.774 & 0.503 & 1.190 & & & & \\
\hline Differentiation & 0.000 & 2.286 & 1.441 & 3.625 & 0.038 & 1.739 & 1.032 & 2.931 \\
\hline $\begin{array}{l}\text { Depth of } \\
\text { invasion }\end{array}$ & 0.014 & 5.775 & 1.420 & 23.496 & & & & \\
\hline \multicolumn{9}{|l|}{$\mathrm{T} 1+\mathrm{T} 2$} \\
\hline \multicolumn{9}{|l|}{$\mathrm{T} 3+\mathrm{T} 4$} \\
\hline $\begin{array}{l}\text { Lymph node } \\
\text { metastasis }\end{array}$ & 0.000 & 2.486 & 1.600 & 3.865 & & & & \\
\hline \multicolumn{9}{|l|}{ No } \\
\hline \multicolumn{9}{|l|}{$\mathrm{N+}$} \\
\hline $\begin{array}{l}\text { Distant } \\
\text { metastasis }\end{array}$ & 0.000 & 6.176 & 3.843 & 9.925 & & & & \\
\hline $\begin{array}{l}\text { TNM stage } \\
\text { I+II } \\
\text { III+IV }\end{array}$ & 0.000 & 3.624 & 2.236 & 5.874 & 0.000 & 2.979 & 1.719 & 5.163 \\
\hline $\begin{array}{l}\text { III+IV } \\
\text { CEA level }\end{array}$ & 0.017 & 1.691 & 1.099 & 2.601 & 0.201 & 1.350 & 0.853 & 2.137 \\
\hline CA19-9 level & 0.100 & 1.550 & 0.919 & 2.615 & & & & \\
\hline $\begin{array}{l}\text { Vessel or } \\
\text { nerve invasion }\end{array}$ & 0.000 & 5.493 & 3.461 & 8.716 & 0.001 & 2.610 & 1.512 & 4.506 \\
\hline $\begin{array}{l}\text { Chemotherapy } \\
\text { or not }\end{array}$ & 0.681 & 1.095 & 0.711 & 1.687 & 0.003 & 2.013 & 1.264 & 3.208 \\
\hline $\begin{array}{l}\text { ZNF460 } \\
\text { expression }\end{array}$ & 0.003 & 1.967 & 1.261 & 3.069 & 0.038 & 1.636 & 1.028 & 2.603 \\
\hline \multicolumn{9}{|l|}{ Low } \\
\hline High & & & & & & & & \\
\hline
\end{tabular}

We also performed univariate and multivariate analyses of various clinical data to assess the prognostic factor. As shown in Tables $2 \& 3$, there are various parameters associated with OS and RFS in colon cancer. Further Cox proportional-hazards regression analysis showed that ZNF460 expression was an independent prognostic factor for OS (Table 2; $\mathrm{HR}=1.636,95 \% \mathrm{CI}: 1.028-2.603 ; \mathrm{P}=0.038)$ and RFS (Table 3; HR: 2.215; 95\% CI: 1.227-3.997; $\mathrm{P}=0.008$ ). Taken together, the data indicated that ZNF460 high expression predicted a worse survival and was an independent prognostic factor for the patients with colon cancer.

\section{High expression of ZNF460 might be associated with lymph node metastasis of colon cancer}

We defined the prognostic value of ZNF460 expression in $\mathrm{N} 0$ stage and $\mathrm{N}+(\mathrm{N} 1, \mathrm{~N} 2)$ stages. The results showed that high expression of ZNF460 associated with worse OS (Fig. 3A, log rank $\mathrm{P}=0.035$ ) in N0 stage, but it had no statistical difference in $\mathrm{N}+$ stages (Fig. 3B, log rank $\mathrm{P}=0.115$ ). These are consistent with the analysis results of TCGA database (N0: Fig. 3C, $\log$ rank $P=0.0053$; N+: Fig. 3D, log rank $\mathrm{P}=0.1317)$. We also found that high ZNF460 expression associated with worse OS in male patients (Fig. 3E, log rank $\mathrm{P}=0.0031$ ), but had no statistical significance in female patients (Fig. 3F, $\log$ rank $\mathrm{P}=$ 0.179 ). However, based on TCGA analysis, high ZNF460 expression was associated with worse OS in 
female instead (Fig. $3 \mathrm{H}, \log$ rank $\mathrm{P}<0.001$ ) but not male patients (Fig. 3G, log rank $\mathrm{P}=0.7343$ ). In conclusion, these results suggested that overexpression of ZNF460 was not only associated with lymph node metastasis of colon cancer, but also with geographical region and race.

\section{Downregulation of ZNF460 represses migration and invasion in colon cancer cells}

To further investigate the functions of ZNF460 in

A

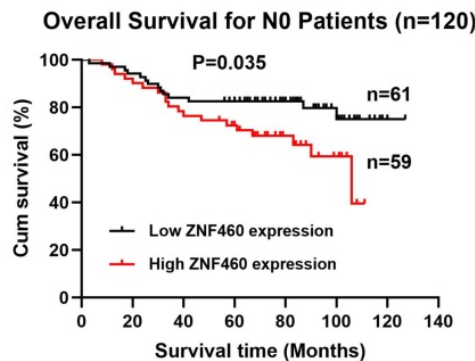

C

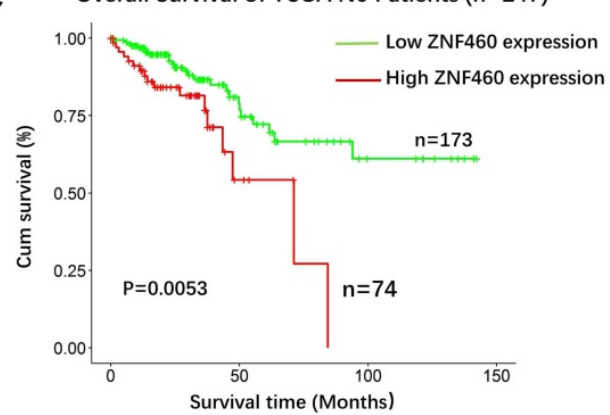

E

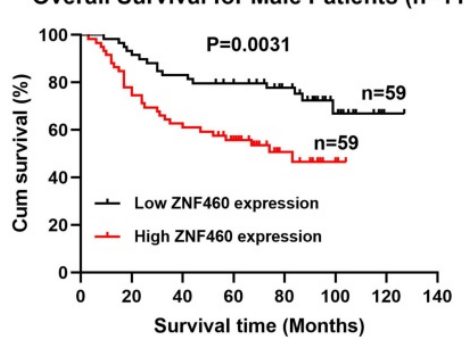

G

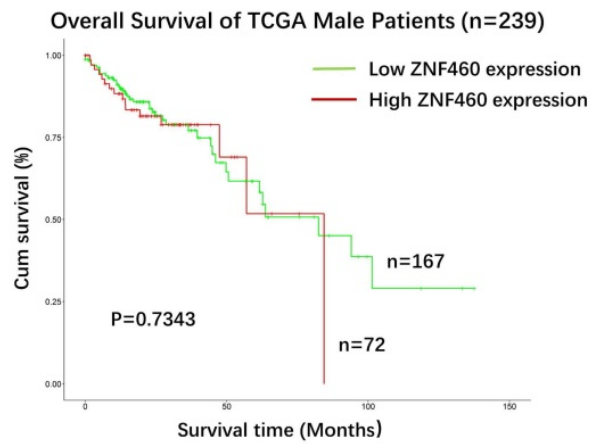

colon cancer cell invasion and migration, we first use WB analyses of ZNF460 expression in the indicated colon cancer cell lines. The results exhibited relatively high expression of ZNF460 among LS174T and LoVo cells (Fig. 4A). Then we manipulated the ZNF460 levels by transfecting ZNF460 siRNA and empty vector siRNA into LS174T (Fig. 4B) and LoVo (Fig. 4C) cells. Finally, we found that ZNF460-siRNA\#1 had the best knockdown effect, which were shown in Fig. 4B and $4 \mathrm{C}$. We found that cells with knockdown of
B

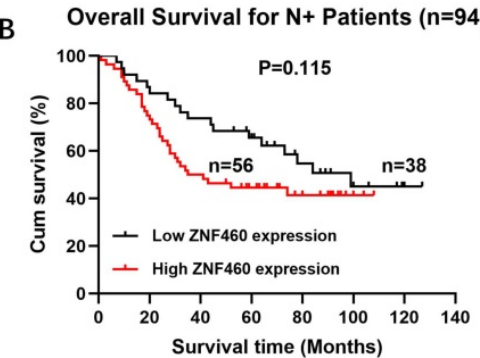

D Overall Survival of TCGA N+ Patients $(n=163)$

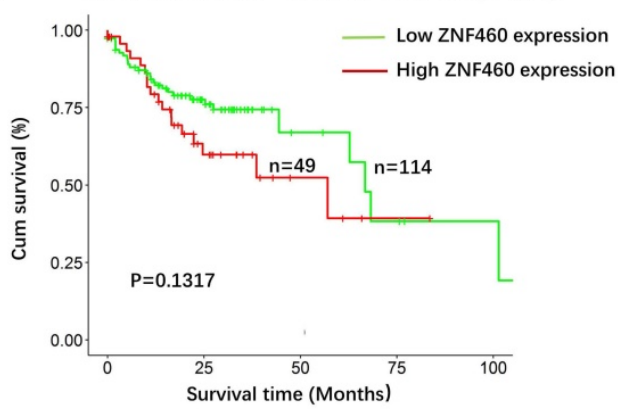

$\mathbf{F}$

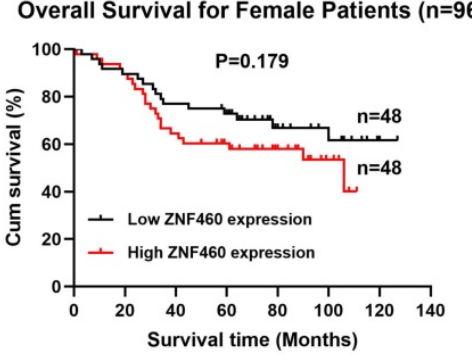

$\mathrm{H}$

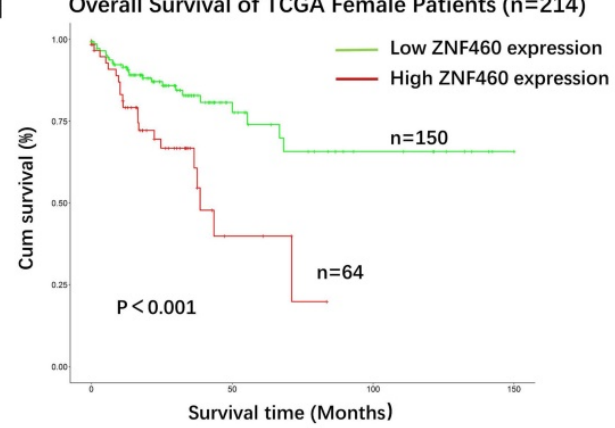

Figure 3. High expression of ZNF460 may be associated with lymph node metastasis of colon cancer. (A) OS for N0 patients. Log rank $\mathrm{P}=0.035$. (B) OS for $\mathrm{N}+$ patients. Log rank $P=0.115$. (C) OS for N0 patients from TCGA database. Log rank $P=0.0053$. (D) OS for $N+$ patients from TCGA database. Log rank $P=0.1317$. (E) OS for male patients. Log rank $P=0.0031$. (F) OS for female patients. Log rank $P=0.179$. (G) OS for male patients from TCGA database. Log rank $P=0.7343$. (H) OS for female patients from TCGA database. Log rank $\mathrm{P}<0.001$. 
ZNF460 displayed a significant decrease in cell migration and invasion abilities compared to cells transfected with the vector control (Fig. 4D).

\section{ZNF460 co-expression networks and GOI KEGG biological process enrichment}

To further explore the mechanism of ZNF460 in promoting the invasion and metastasis of colon cancer, LinkedOmics was used to examine ZNF460 co-expression genes in colon cancer cohort. The top 50 significant genes positively and negatively correlated with ZNF460 were shown in the heatmap (Figure 5A, $\mathrm{B})$. The gene with the strongest positive correlation with the expression of ZNF460 was ZNF484, and the strongest negative correlation was MGAT4B.
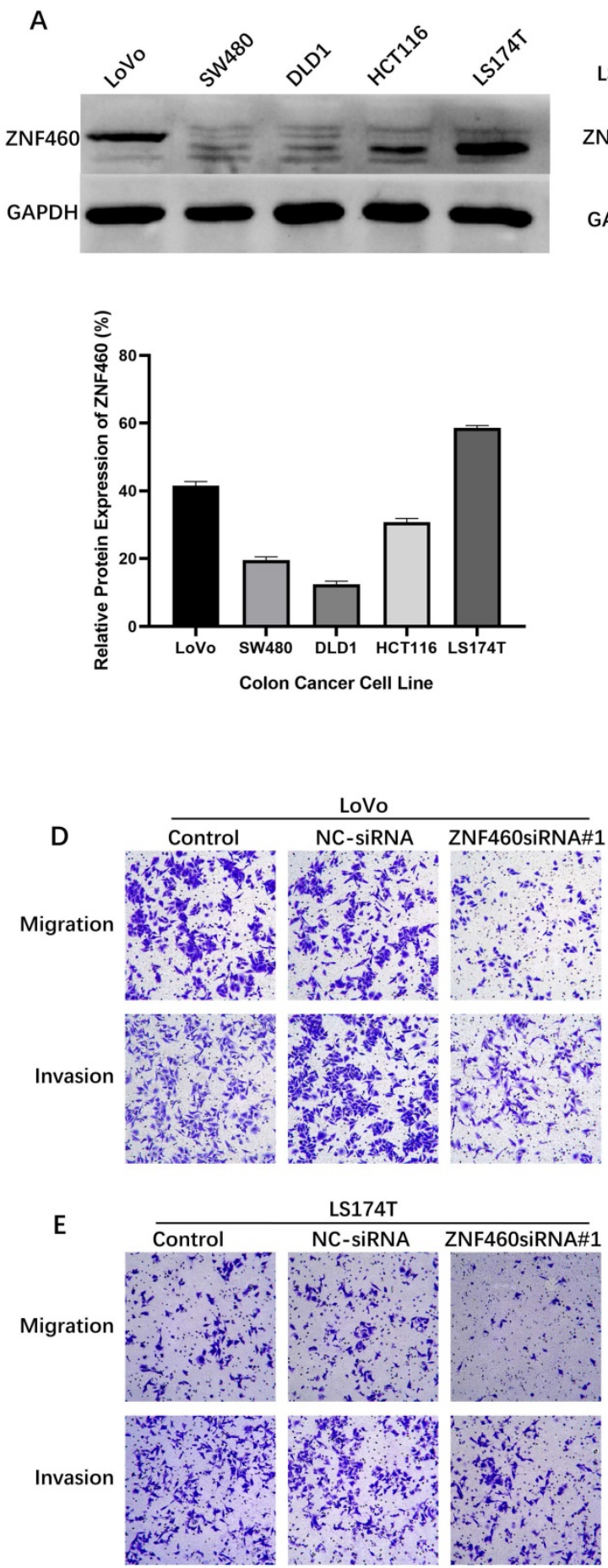
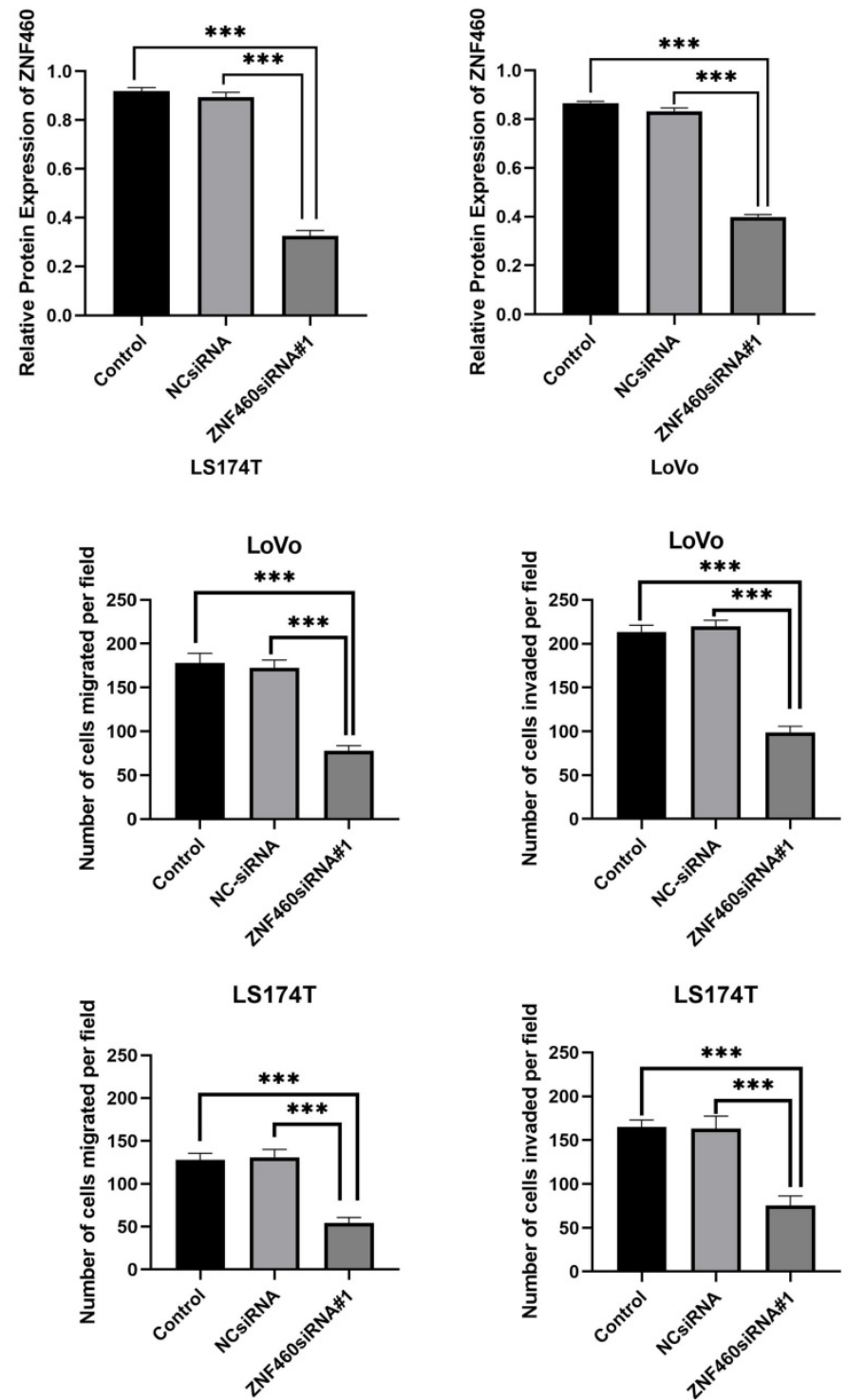

Figure 4. Downregulation of ZNF460 represses migration and invasion in colon cancer cells. (A) Expression level of ZNF460 in colon cancer cells. (B,C) LS174T and LoVo transfected with ZNF460siRNA\#1. *** P<0.001. (D) Alteration of LoVo migration and invasion ability. ${ }^{* * *} \mathrm{P}<0.001$. (E) Alteration of LS174T migration and invasion ability. $* * * \mathrm{P}<0.001$. 
ZNF460 Correlated Significant Genes (Pearson test)

A

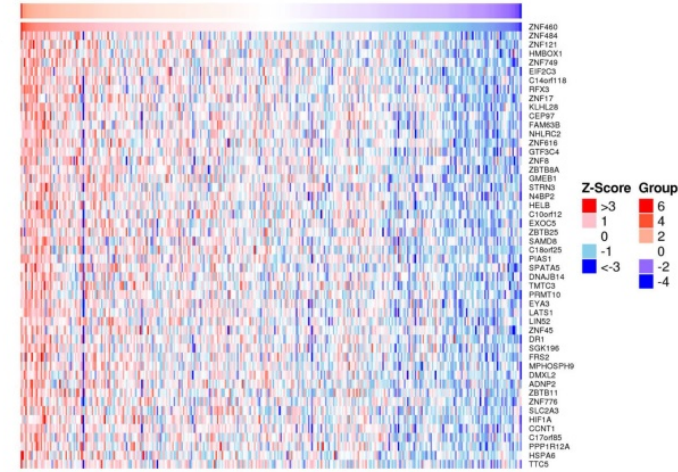

B

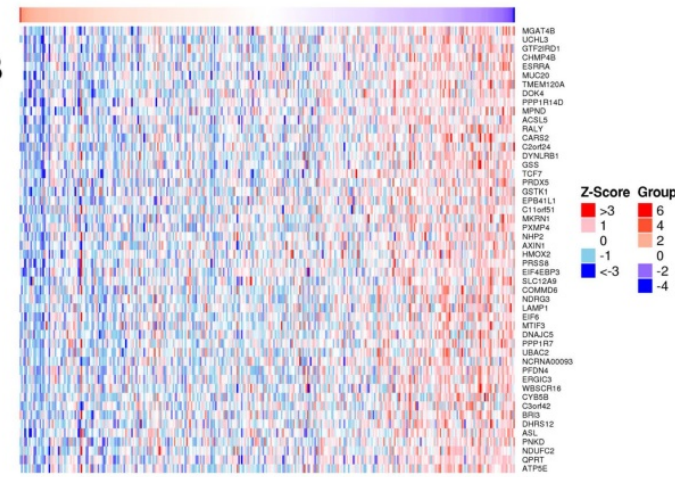

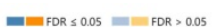
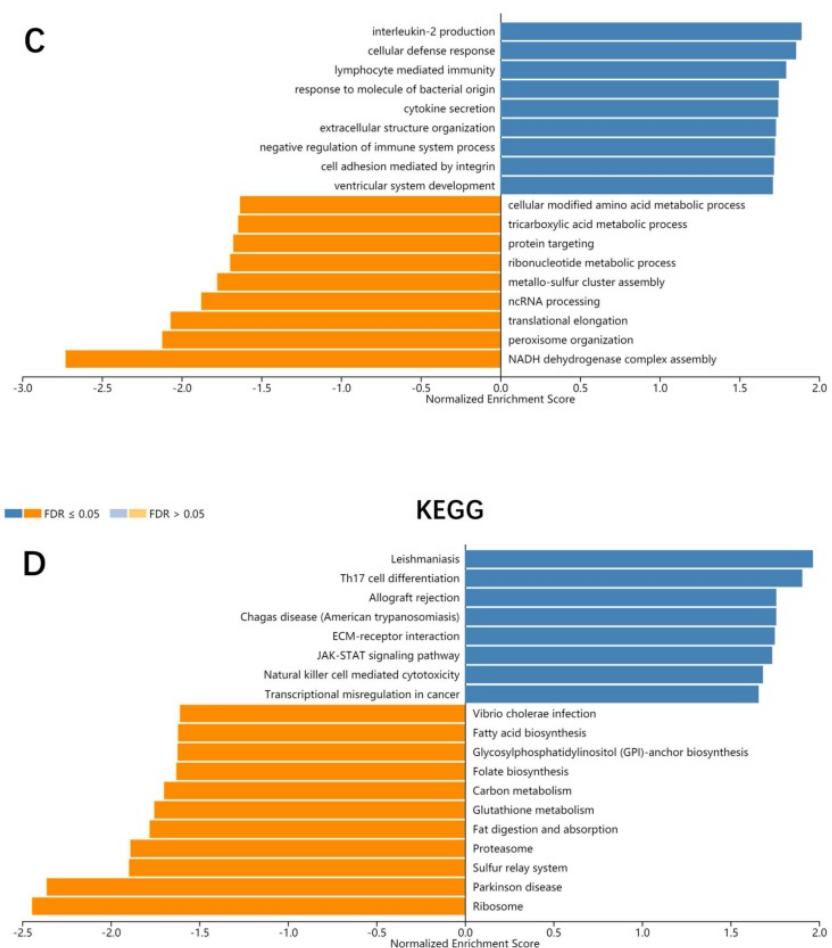

Figure 5. ZNF460 co-expression genes in colon cancer (LinkedOmics). (A,B) Heatmaps showing top 50 genes positively and negatively correlated with ZNF460 in colon cancer cohort. Red indicates positively correlated genes and blue indicates negatively correlated genes. (C,D) Significantly enriched GO annotations and KEGG pathways of ZNF460 in colon cancer cohort.

Table 3. Cox proportional-hazard regression analysis for Recurrence Free Survival in 180 patients with colon cancer

\begin{tabular}{|c|c|c|c|c|c|c|c|c|}
\hline \multirow[t]{3}{*}{ Characteristic } & \multicolumn{4}{|c|}{ Univariate analysis } & \multicolumn{4}{|c|}{ Multivariate analysis } \\
\hline & \multirow[t]{2}{*}{$P$-value } & \multirow[t]{2}{*}{ HR } & \multicolumn{2}{|c|}{$\begin{array}{l}95.0 \% \text { CI for } \\
\operatorname{Exp}(\mathrm{B})\end{array}$} & \multirow{2}{*}{\multicolumn{2}{|c|}{$P$-value HR }} & \multicolumn{2}{|c|}{$\begin{array}{l}95.0 \% \text { CI for } \\
\operatorname{Exp}(B)\end{array}$} \\
\hline & & & Lower & Upper & & & Lower & Upper \\
\hline Gender & 0.186 & 1.458 & 0.834 & 2.550 & & & & \\
\hline Age & 0.720 & 0.903 & 0.519 & 1.574 & & & & \\
\hline $\begin{array}{l}\text { Tumor } \\
\text { location }\end{array}$ & 0.902 & 0.966 & 0.552 & 1.688 & & & & \\
\hline Differentiation & 0.009 & 2.234 & 1.219 & 4.095 & 0.083 & 1.892 & 0.921 & 3.889 \\
\hline $\begin{array}{l}\text { Depth of } \\
\text { invasion } \\
\mathrm{T} 1+\mathrm{T} 2 \\
\mathrm{~T} 3+\mathrm{T} 4\end{array}$ & 0.039 & 8.019 & 1.107 & 58.078 & & & & \\
\hline $\begin{array}{l}\text { Lymph node } \\
\text { metastasis }\end{array}$ & 0.007 & 2.163 & 1.236 & 3.785 & & & & \\
\hline No & & & & & & & & \\
\hline $\mathrm{N}+$ & & & & & & & & \\
\hline $\begin{array}{l}\text { TNM stage } \\
\text { I+II }\end{array}$ & 0.007 & 2.163 & 1.236 & 3.785 & 0.109 & 1.654 & 0.893 & 3.061 \\
\hline III & & & & & & & & \\
\hline CEA level & 0.025 & 1.885 & 1.083 & 3.284 & 0.040 & 1.807 & 1.029 & 3.175 \\
\hline CA19-9 level & 0.679 & 1.173 & 0.551 & 2.500 & & & & \\
\hline $\begin{array}{l}\text { Vessel or } \\
\text { nerve invasion }\end{array}$ & 0.001 & 3.613 & 1.689 & 7.726 & 0.268 & 1.671 & 0.673 & 4.149 \\
\hline $\begin{array}{l}\text { Chemotherapy } \\
\text { or not }\end{array}$ & 0.288 & 1.354 & 0.774 & 2.368 & 0.662 & 1.143 & 0.627 & 2.085 \\
\hline $\begin{array}{l}\text { ZNF460 } \\
\text { expression }\end{array}$ & 0.004 & 2.321 & 1.300 & 4.141 & 0.008 & 2.215 & 1.227 & 3.997 \\
\hline Low & & & & & & & & \\
\hline High & & & & & & & & \\
\hline
\end{tabular}

Significant Gene Ontology (GO) term annotation by gene set enrichment analysis [17] (GSEA) showed that ZNF460 co-expressed genes participate primarily in interleukin-2 production, cellular defense response, and lymphocyte mediated immunity, while the NADH dehydrogenase complex assembly, and peroxisome organization were inhibited (Figure 5C). Kyoto Encyclopedia of Genes and Genomes (KEGG) pathway analysis showed enrichment in the ECM-receptor, and JAK-STAT signaling pathway, etc. (Figure 5D). These results showed that ZNF460 played an extensive role in the progression of colon cancer.

\section{ZNF460 promotes metastasis through JAK2/ STAT3 signaling pathway}

According to the results of bioinformatics analysis, ZNF460 may promote the metastasis of colon cancer by activating the JAK/STAT signaling pathway. To further elucidate the mechanism, western blot was utilized to detect protein expression of p-JAK2, JAK2, p-STAT3 and STAT3 in colon cancer cells after transfection with ZNF460 siRNA. The results showed that downregulation of ZNF460 expression significantly decreased the p-JAK2 and p-STAT3 expression levels in colon cancer cells (Figure 6). This result showed that ZNF460 promotes metastasis through JAK2/STAT3 signaling pathway. 

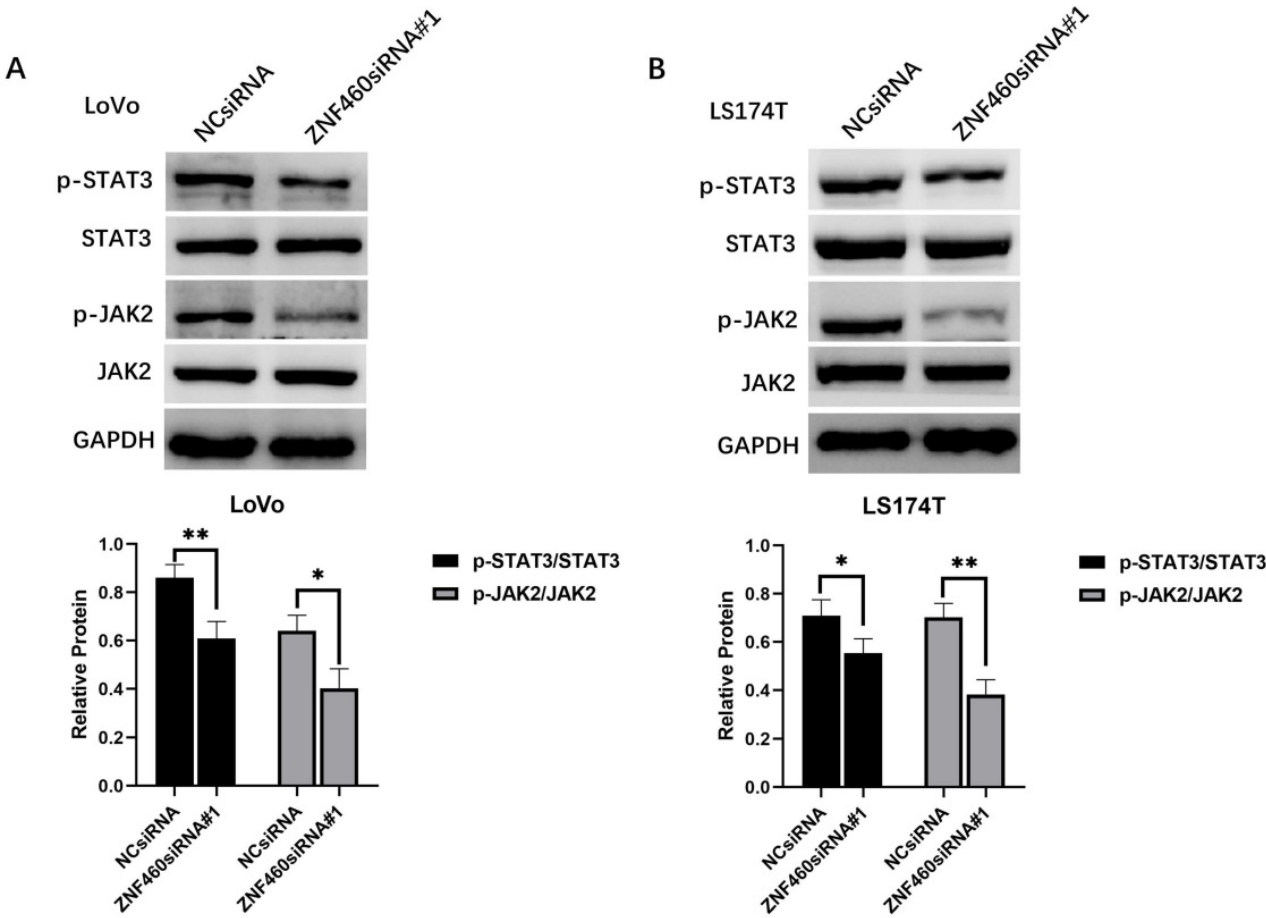

Figure 6. ZNF460 promotes metastasis through JAK2/STAT3 signaling pathway. (A) Expression level of $\mathrm{p}-\mathrm{STAT3} / \mathrm{STAT} 3$ and $\mathrm{p}-\mathrm{JAK} 2 / \mathrm{JAK} 2$ in LoVo. * P<0.05, $* *$ P $<0.01$. (B) Expression level of $\mathrm{P}-\mathrm{STAT} 3 / \mathrm{STAT} 3$ and $\mathrm{P}-\mathrm{JAK} 2 / \mathrm{JAK} 2$ in LS174T. $* \mathrm{P}<0.05, * * \mathrm{P}<0.01$.

\section{Discussion}

ZNF460 contains a KRAB A+B box and eleven $\mathrm{C} 2 \mathrm{H} 2$ type zinc finger motifs which was first described in 2003 [15]. Previous studies had demonstrated that ZNFs could recruit different chromatin modifiers and served diverse regulation mechanisms. For example, ZNFs could recruit co-repressors and play a role of transcriptional repressor [19]. On the other hand, it could work as transcriptional activators by interacting with co-activators, including CBP/p300 and C/EBP [20]. However, the expression of ZNF460 in colon cancer and its function in the process of colon cancer invasion and metastasis remained unclear. In this study, we first showed that ZNF460 was overexpressed in colon cancer specimens, was significantly correlated with poor survival, and promoted the invasion and metastasis of colon cancer. Multivariate Cox regression analysis demonstrated ZNF460 expression to be an independent prognostic factor for OS and RFS in colon cancer patients. Mechanistically, we revealed that ZNF460 promotes metastasis through JAK2/STAT3 signaling pathway in colon cancer cells. These results confirmed that ZNF460 was a potential prognostic biomarker and therapeutic target in patients with colon cancer.

Previous studies had reported that several ZNFs were associated with tumor prognosis. To be exact, the zinc finger and BTB domain-containing 7C (ZBTB7C) had been reported to be downregulated in colorectal cancer and could act as an independent prognostic factor [21]. The Zinc-fingers and homeoboxes 3 (ZHX3) and ZNF93 had been reported to be significantly increased and were associated with poor prognosis in gastric cancer [22] and ovarian cancer [23], respectively. Consistent with results of previous studies, our study showed for the first time that ZNF460 expression was significantly upregulated in colon cancer tissues at both the mRNA and protein levels, which was associated with poor prognosis and could be an independent prognostic factor in colon cancer patients. We also found that high expression of ZNF460 had a worse prognosis in male than female. This result was contradictory to the TCGA result, suggesting that it might be related to geography and race.

In recent years, more and more ZNFs were identified as oncogene and promoted various tumors via enhancing cell proliferation [24], promoting cell migration and invasion [25], suppressing autophagy [26], and epithelial-mesenchymal transition [27]. ZNF460 gene co-expression network analysis established that ZNF460 had a positive correlation with ZNF484, ZNF121, HMBOX1 and a negative correlation with MGAT4B, UCHL3, GTF2IRD1. It had been confirmed that ZNF121 and HMBOX1 played a role in promoting cancer proliferation in breast cancer and gastric cancer $[28,29]$. Therefore, we speculated that ZNF121 and HMBOX1 played a collaborative role with ZNF460 in carcinogenesis of colon cancer. 
Many studies had confirmed that ZNFs family promoted the progression of colorectal cancer. Xing et al. found that ZNF692 promoted colon adenocarcinoma cell growth and metastasis by activating the PI3K/AKT pathway [30], and Qin et al. also found that ZNF281 regulates cell proliferation, migration and invasion in colorectal cancer through Wnt/ $\beta$-Catenin signaling [31]. Consistent with previous studies, our study also indicated ZNF460 promoted colon cancer invasion and migration, especially lymph node metastasis. To further explore the mechanism of ZNF460 in promoting the invasion and metastasis of colon cancer, we found that ZNF460 activated JAK2/STAT3 signaling pathway. These results suggested that ZNF460 promoted invasion and metastasis by activating the JAK-STAT signaling pathway. Similar to our results, Daniel Triner et al. reported that myc-associated zinc finger (MAZ) protein regulated the proinflammatory response in colon cancer via STAT3 signaling [32], and Vikas Verma et al. reported that the activity of JAK-STAT signaling pathway was affected by artificially altering the expression level of ZNF143 [33]. Zeng YT et al. also found that ZFP42 zinc finger protein promoted metastasis of cervical cancer by upregulating the activity of the JAK2/STAT3 pathway [34].

In summary, we found that ZNF460 was overexpressed in colon cancer, and its expression was correlated with clinical outcome. ZNF460 overexpression was significantly associated with poor prognosis and increased metastatic capabilities of human colon cancer by activating JAK2/STAT3 signaling pathway, and ZNF460 might act as a potential prognostic biomarker and therapeutic target.

\section{Acknowledgements}

This work was supported by the Sanming Project of Medicine in Shenzhen, Guangdong Province, China (SZSM201612022 to ZL and YW), The Shenzhen Key Medical Discipline Construction Fund Guangdong Province, China (No. SZXK016).

\section{Competing Interests}

The authors have declared that no competing interest exists.

\section{References}

1. Bray F, Ferlay J, Soerjomataram I, Siegel RL, Torre LA, Jemal A. Global cancer statistics 2018: GLOBOCAN estimates of incidence and mortality worldwide for 36 cancers in 185 countries. CA: a cancer journal for clinicians. 2018; 68: 394-424.

2. Dekker E, Tanis PJ, Vleugels JLA, Kasi PM, Wallace MB. Colorectal cancer. Lancet (London, England). 2019; 394: 1467-80.

3. Argilés G, Tabernero J, Labianca R, Hochhauser D, Salazar R, Iveson T, et al. Localised colon cancer: ESMO Clinical Practice Guidelines for diagnosis, treatment and follow-up. Annals of oncology : official journal of the European Society for Medical Oncology. 2020; 31: 1291-305.
4. Li J, Yuan Y, Yang F, Wang Y, Zhu X, Wang Z, et al. Expert consensus on multidisciplinary therapy of colorectal cancer with lung metastases (2019 edition). Journal of hematology \& oncology. 2019; 12: 16.

5. Zhou W, Yang F, Peng J, Wang F, Lin Y, Jiang W, et al. High pretreatment serum CA19-9 level predicts a poor prognosis for patients with stage III colon cancer after curative resection and adjuvant chemotherapy. Journal of Cancer. 2019; 10: 3810-8.

6. Wang Z, Wang X, Yuan J, Zhang X, Zhou J, Lu M, et al. Survival Benefit of Palliative Local Treatments and Efficacy of Different Pharmacotherapies in Colorectal Cancer With Lung Metastasis: Results From a Large Retrospective Study. Clinical colorectal cancer. 2018; 17: e233-e55.

7. Ding L, Lan Z, Xiong X, Ao H, Feng Y, Gu H, et al. The Dual Role of MicroRNAs in Colorectal Cancer Progression. International journal of molecular sciences. 2018; 19.

8. Valastyan S, Weinberg RA. Tumor metastasis: molecular insights and evolving paradigms. Cell. 2011; 147: 275-92.

9. Lander ES, Linton LM, Birren B, Nusbaum C, Zody MC, Baldwin J, et al. Initial sequencing and analysis of the human genome. Nature. 2001; 409: 860-921.

10. Jen J, Wang YC. Zinc finger proteins in cancer progression. Journal of biomedical science. 2016; 23: 53 .

11. Yang L, Hamilton SR, Sood A, Kuwai T, Ellis L, Sanguino A, et al. The previously undescribed ZKSCAN3 (ZNF306) is a novel "driver" of colorectal cancer progression. Cancer research. 2008; 68: 4321-30.

12. Serra RW, Fang M, Park SM, Hutchinson L, Green MR. A KRAS-directed transcriptional silencing pathway that mediates the $\mathrm{CpG}$ island methylator phenotype. eLife. 2014; 3: e02313.

13. Postigo AA, Depp JL, Taylor JJ, Kroll KL. Regulation of Smad signaling through a differential recruitment of coactivators and corepressors by ZEB proteins. The EMBO journal. 2003; 22: 2453-62.

14. Cheng Y, Liang P, Geng H, Wang Z, Li L, Cheng SH, et al. A novel 19q13 nucleolar zinc finger protein suppresses tumor cell growth through inhibiting ribosome biogenesis and inducing apoptosis but is frequently silenced in multiple carcinomas. Molecular cancer research : MCR. 2012; 10: 925-36.

15. Dai J, Li Y, Ji C, Jin F, Zheng Z, Wang X, et al. Characterization of two novel KRAB-domain-containing zinc finger genes, ZNF460 and ZNF461, on human chromosome 19q13.1-->q13.4. Cytogenetic and genome research. 2003; 103: 74-8.

16. Budwit-Novotny DA, McCarty KS, Cox EB, Soper JT, Mutch DG, Creasman WT, et al. Immunohistochemical analyses of estrogen receptor in endometrial adenocarcinoma using a monoclonal antibody. Cancer research. 1986; 46: 5419-25.

17. Chandrashekar DS, Bashel B, Balasubramanya SAH, Creighton CJ, Ponce-Rodriguez I, Chakravarthi B, et al. UALCAN: A Portal for Facilitating Tumor Subgroup Gene Expression and Survival Analyses. Neoplasia (New York, NY). 2017; 19: 649-58.

18. Vasaikar SV, Straub P, Wang J, Zhang B. LinkedOmics: analyzing multi-omics data within and across 32 cancer types. Nucleic acids research. 2018; 46: D956-d63.

19. Frietze S, O'Geen H, Blahnik KR, Jin VX, Farnham PJ. ZNF274 recruits the histone methyltransferase SETDB1 to the $3^{\prime}$ ends of ZNF genes. PloS one. 2010; 5: e15082.

20. Jeon BN, Kim MK, Yoon JH, Kim MY, An H, Noh HJ, et al. Two ZNF509 (ZBTB49) isoforms induce cell-cycle arrest by activating transcription of p21/CDKN1A and RB upon exposure to genotoxic stress. Nucleic acids research. 2014; 42: 11447-61.

21. Chen $X$, Jiang $Z, P u ~ Y$, Jiang $X$, Xiang L, Jiang Z. Zinc finger and BTB domain-containing 7C (ZBTB7C) expression as an independent prognostic factor for colorectal cancer and its relevant molecular mechanisms. American journal of translational research. 2020; 12: 4141-59.

22. You Y, Bai F, Li H, Ma Y, Yao L, Hu J, et al. Prognostic value and therapeutic implications of ZHX family member expression in human gastric cancer. American journal of translational research. 2020; 12: 3376-88.

23. Cui XX, Zhou C, Lu H, Han YL, Wang FM, Fan WR, et al. High expression of ZNF93 promotes proliferation and migration of ovarian cancer cells and relates to poor prognosis. International journal of clinical and experimental pathology. 2020; 13: 944-53.

24. Zhang Y, Li Q, Wei S, Sun J, Zhang X, He L, et al. ZNF143 Suppresses Cell Apoptosis and Promotes Proliferation in Gastric Cancer via ROS/p53 Axis. Disease markers. 2020; 2020: 5863178 .

25. Cassandri M, Smirnov A, Novelli F, Pitolli C, Agostini M, Malewicz M, et al. Zinc-finger proteins in health and disease. Cell death discovery. 2017; 3: 17071.

26. Barthez M, Poplineau M, Elrefaey M, Caruso N, Graba Y, Saurin AJ. Human ZKSCAN3 and Drosophila M1BP are functionally homologous transcription factors in autophagy regulation. Scientific reports. 2020; 10: 9653.

27. Wang $\mathrm{H}, \mathrm{Xu} \mathrm{H}, \mathrm{Ma} \mathrm{F}$, Zhan $\mathrm{M}$, Yang $\mathrm{X}$, Hua S, et al. Zinc finger protein 703 induces EMT and sorafenib resistance in hepatocellular carcinoma by transactivating CLDN4 expression. Cell death \& disease. 2020; 11: 225.

28. Luo A, Zhang X, Fu L, Zhu Z, Dong JT. Zinc finger factor ZNF121 is a MYC-interacting protein functionally affecting MYC and cell proliferation in epithelial cells. Journal of genetics and genomics = Yi chuan xue bao. 2016; 43: 677-85.

29. Diao N, Li Y, Yang J, Jin C, Meng X, Jiao W, et al. High expression of HMBOX1 contributes to poor prognosis of gastric cancer by promoting cell proliferation and migration. Biomedicine \& pharmacotherapy $=$ Biomedecine \& pharmacotherapie. 2019; 115: 108867. 
30. Xing Y, Ren S, Ai L, Sun W, Zhao Z, Jiang F, et al. ZNF692 promotes colon adenocarcinoma cell growth and metastasis by activating the PI3K/AKT pathway. International journal of oncology. 2019; 54: 1691-703.

31. Qin CJ, Bu PL, Zhang Q, Chen JT, Li QY, Liu JT, et al. ZNF281 Regulates Cell Proliferation, Migration and Invasion in Colorectal Cancer through Wnt/ $\beta$-Catenin Signaling. Cellular physiology and biochemistry: international journal of experimental cellular physiology, biochemistry, and pharmacology. 2019; 52: 1503-16.

32. Triner D, Castillo C, Hakim JB, Xue X, Greenson JK, Nuñez G, et al. MycAssociated Zinc Finger Protein Regulates the Proinflammatory Response in Colitis and Colon Cancer via STAT3 Signaling. Molecular and cellular biology. $2018 ; 38$.

33. Verma V, Paek AR, Choi BK, Hong EK, You HJ. Loss of zinc-finger protein 143 contributes to tumour progression by interleukin-8-CXCR axis in colon cancer. Journal of cellular and molecular medicine. 2019; 23: 4043-53.

34. Zeng YT, Liu XF, Yang WT, Zheng PS. REX1 promotes EMT-induced cell metastasis by activating the JAK2/STAT3-signaling pathway by targeting SOCS1 in cervical cancer. Oncogene. 2019; 38: 6940-57. 